\title{
Exercise training combined with electromyostimulation in the rehabilitation of patients with chronic heart failure: $A$ randomized trial
}

\author{
Vladimir Soskaa, Petr Dobsak ${ }^{b}$, Michal Pohanka $^{b}$, Lenka Spinarovac, Jiri Vitovecc, Jan Krejcic, Petr Hudec, Pavel Homolka ${ }^{b}$, \\ Marie Novakovad ${ }^{d}$ Jean-Christophe Eicher ${ }^{e}$, Jean-Eric Wolfe, Ladislav Dusek ${ }^{f}$, Jarmila Siegelova ${ }^{b}$
}

\begin{abstract}
Aim. Both aerobic training (AT) and electromyostimulation (EMS) of leg muscles improve exercise tolerance in patients suffering from chronic heart failure (CHF). It was speculated that combination of both methods might have an additive effect. This study was performed to evaluate the effects of a combination of AT and EMS in rehabilitation (RHB) of CHF patients.

Patients and Methods. Patients ( $n=71$; age 59 \pm 10.2 yrs, NYHA II/III, EF 32 $\pm 7.1 \%$ ) were randomized into 3 groups: a) group AT, b) group EMS, and c) group AT+EMS. AT protocol included standard activity on bicycle $3 x$ a week at the level of individual anaerobic threshold. EMS (10Hz, mode $20 \mathrm{~s}$ "on"/20s "off") was applied to leg extensors for $2 \mathrm{~h} /$ day. Total time of given type of RHB was 12 weeks.

Results. Data analysis revealed statistically significant improvements of patients in all experimental groups (averaged difference after 12 weeks of exercise as related to initial value: $\left.\Delta \mathrm{VO}_{2 \text { peak }}:+12.9 \%, \Delta \mathrm{VO}_{2 \mathrm{AT}}:+9.3 \%, \Delta \mathrm{W}_{\text {peak }}:+22.7 \%\right)$. No statistically significant difference among experimental groups was found. Quality of life (Minnesota Living with Heart Failure - MLHF) global score was significantly improved in all 3 groups: AT ( $\triangle M L H F:-27.9 \% ; P=0.001)$, AT+EMS $(\triangle M L H F$ : -29.1\%; $P=0.002$ ), and EMS ( $\triangle$ MLHF: $-16.6 \% ; P=0.008$ ). MLHF score in EMS group showed the smallest time-related improvement compared to AT and AT+EMS groups, and this difference in improvement between the groups was statistically significant $(P=0.021)$.

Conclusion. No significant difference was found between the two types of exercise training.and nor did, their combination have any significant additional improvement.
\end{abstract}

Key words: exercise, electromyostimulation, heart failure, quality of life, combined training

Received: February 27, 2012; Accepted with revision: October 11, 2012; Available online: November 1, 2012 http://dx.doi.org/10.5507/bp.2012.096

a'Department of Clinical Biochemistry, St. Anne's University Hospital in Brno and Masaryk University, Brno, Czech Republic
'Department of Sports Medicine and Rehabilitation, St. Anne's University Hospital in Brno and Masaryk University, Brno
c $7^{\text {st }}$ Department of Internal Medicine, St. Anne's University Hospital in Brno and Masaryk University, Brno
${ }^{d}$ Institute of Physiology, Faculty of Medicine, Masaryk University, Brno
eDepartment of Cardiology II, Hospital du Bocage and University of Burgundy, Dijon, France
IInstitute of Biostatistics and Analyses, Faculty of Medicine, Masaryk University, Brno
Corresponding author: Petr Dobsak, e-mail: petr.dobsak@fnusa.cz

\section{INTRODUCTION}

Congestive chronic heart failure (CHF) is a frequent, gradually progressing and life-threatening disease occurring in $2 \%$ of the adult population in developed countries. Its incidence increases to $6-10 \%$ for populations above 65 years of age ${ }^{1}$. General deconditioning and the functional limitations in $\mathrm{CHF}$ are the results of the increasing number of pathophysiological changes caused by ventricular dysfunction and peripheral changes ${ }^{2,3}$. These peripheral changes include extensive morphological and functional abnormalities of skeletal muscles, especially loss of muscle mass, decreased number of type I (slow fibers), and reduced terminal microcirculation density ${ }^{4,5}$. At the metabolic level, muscular abnormalities are characterized by a decrease in the number of mitochondria and decline in their oxidation capacity ${ }^{6-8}$. Current treatment of heart failure (CHF) encompasses not only appropriate pharmacotherapy but also meticulous education consisting in changing patient's life style and performing adequate physical activity 9 . These measures are inseparably related to cardiovascular rehabilitation (RHB), which effectively contributes to optimizing the entire treatment. Thus, RHB represents today the standard non-pharmacological strategy and aerobic exercise training (ET) is a generally accepted tool for improving exercise tolerance and CHF patients' prognosis ${ }^{10-12}$. The first reports on the use of electromyostimulation (EMS) for RHB in CHF patients appeared almost 15 years $\mathrm{ago}^{13}$; at present, there are sufficient arguments for using EMS as an alternative to classical training, in particular in patients with advanced stages of CHF (ref. ${ }^{14}$ ). EMS and voluntary muscular contractions (VMC) during bicycle training are two different methods of activating muscle fibers. They both cause a number of acute changes at the level of the neuromuscular system (exercise training on bicycle is a dynamic exercise, while EMS is a rather static exercise). Therefore, long-term application of either type of muscle activation causes various types of resulting muscular adaptation. It can be presumed that using the combination of 
both types of exercise may lead to cumulative adaptation in the given muscle, as well as to higher overall efficiency. This presumption is confirmed by a few studies on the combination of EMS and VMC, in the area of muscle rehabilitation and regeneration after surgery ${ }^{15,16}$. From this perspective, achieving a higher training benefit for CHF patients would be of great importance, but to date the effects of ET + EMS combination in these patients have not been reported. The main purpose of this study was to evaluate the effects of the combination of standard aerobic training (AT) and the low-frequency EMS in the rehabilitation of patients with $\mathrm{CHF}$ of moderate grade.

\section{PATIENTS AND METHODS}

Before inclusion into the study, all patients underwent a spiroergometric test to evaluate their cardiopulmonary performance. Peak oxygen consumption $\left(\mathrm{VO}_{2 \text { peak }}\right)$ was measured during gradually increasing load using a spiroergometric system (Power Cube, Ganshorn ${ }^{\circledR}$ Medizin Electronic, Niederlauer, Germany) with an integrated 12-lead ECG (AT-104 PC, Schiller ${ }^{\circledR}$, Baar, Switzerland) and a bicycle ergometer (Ergoselect 200, Ergoline ${ }^{\circledR}$, Bitz, Germany). Spiroergometric test was done up to the peak of individual functional capacity. A ramp protocol was used. Patients were instructed to maintain a stable RPM (60 rev. $\mathrm{min}^{-1}$ ) and the workload was automatically increased gradually from 0 to the tolerated maximum (W.min ${ }^{-1}$ ). HR was continuously and automatically recorded during the test, and BP was measured manually every $2 \mathrm{~min}$. Standard ventilation and respiratory gas exchange parameters $\left(\mathrm{VO}_{2}, \mathrm{VCO}_{2}\right.$, ventilation - VE) were measured using the "breath by breath" method, $\mathrm{VO}_{2 \text { peak }}$ was expressed as the highest value of $\mathrm{O}_{2}$ reached in the last 30 s of exercise. Peak HR $\left(\mathrm{HR}_{\text {peak }}\right)$ and peak workload $\left(\mathrm{W}_{\text {peak }}\right)$ were determined in the same way. Anaerobic threshold $\left(\mathrm{VO}_{2 \mathrm{AT}}\right)$ was determined by a standard method according to Wasserman ${ }^{17}$. The spiroergometer was calibrated individually for each patient. Each test was performed by a physician and a specially trained nurse. An identical protocol for the spiroergometric test was also used after completion of the RHB program. Eighty-six patients with moderate $\mathrm{CHF}$ were enrolled. After recruitment and initial testing, eligible patients signed the informed consent. Patients were randomly assigned to 3 groups: a) group with aerobic training (AT); b) group with electromyostimulation (EMS); and c) group with combination of aerobic training and electromyostimulation (AT+EMS). The experimental design including randomization plan was set up in statistical package SPSS (IBM Corporation, 2008). The final randomization procedure ( $3 \mathrm{arms}$, complete random selection of cases) was customized in a local table processor as random number generator. The study protocol was approved by the local Ethics Committee and conformed to the principles outlined in the Declaration of Helsinki (revised 2008 in Seoul) and to the GCP guidelines of the European Community.

Inclusion criteria: symptomatic stability, NYHA II-III, coronary stenosis identified by angiography, LVEF $<40 \%$, and optimized pharmacological treatment (unchanged for 2 months before and throughout the study). Exclusion criteria: unstable angina pectoris, cardiac pacemaker, evolutive ventricular dysrhythmia, intermittent claudication, diabetes mellitus, and chronic broncho-pulmonary disease.

Patients in the AT group $(n=29)$ completed a standard AT on electromagnetically braked bicycle ergometers (REHA E900, Ergoline ${ }^{\circledR}$, Bitz, Germany) controlled by computer program (ErgoSoft ${ }^{\circledR} A G$, Switzerland). The

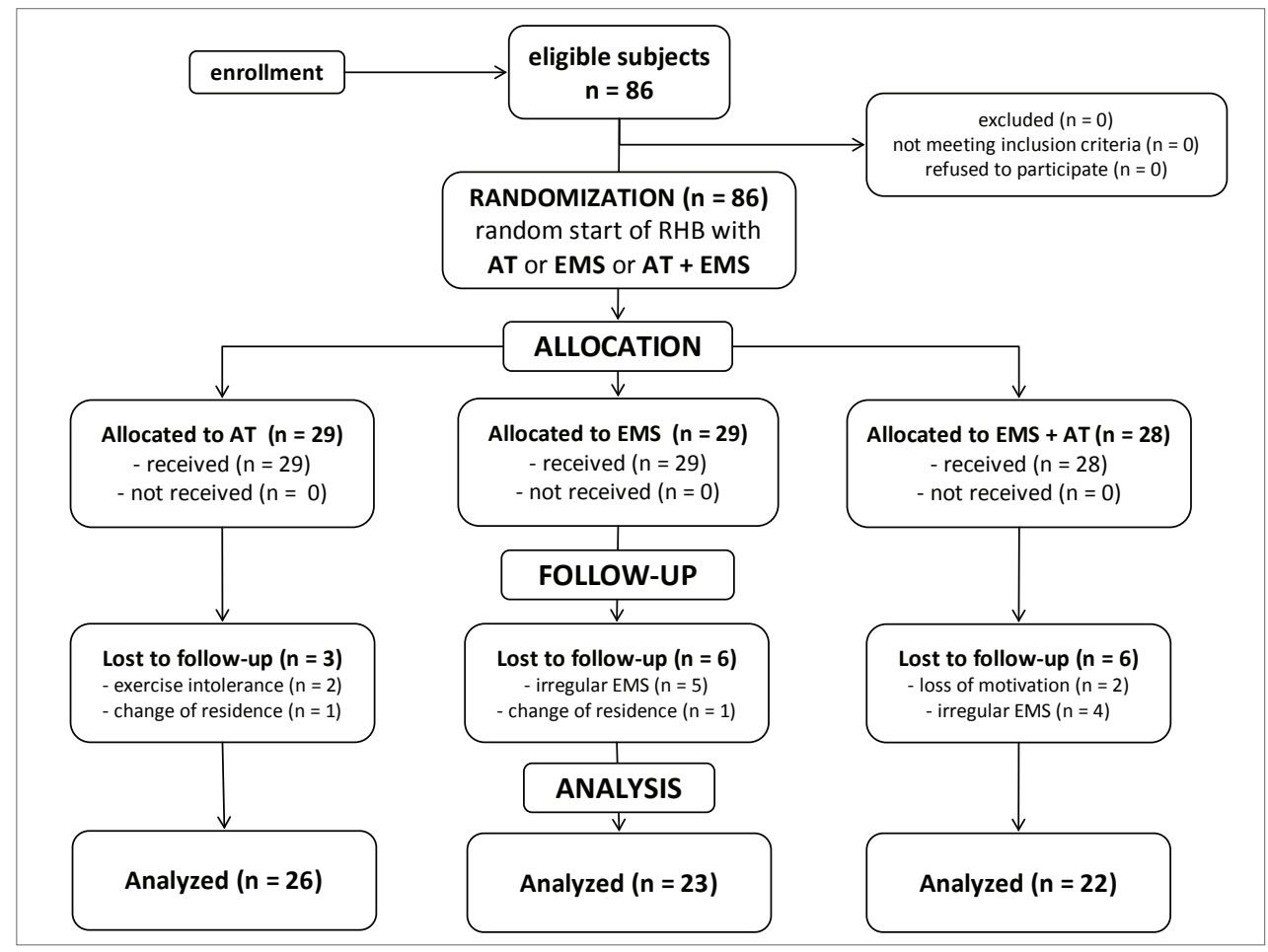

Fig. 1. Flow chart showing enrollment and the randomization procedure. 
training protocol included an initial warm-up phase $(10$ min), interval AT (40 min) and a cool-down phase (10 min). Interval training alternate pedaling with the determined load (1 min) and pedaling without load $(2 \mathrm{~min})$. This training protocol was performed only during the initial 2 weeks. During the following 10 weeks, interval AT was shortened to $20 \mathrm{~min}$ and $20 \mathrm{~min}$ of resistance training (RT) were added. RT included 3 standard exercises: pulley lifting, leg extension and bench press. Load intensity for RT was always determined individually using 1-RM (one repetition maximum) test. Before RT, all patients completed a practice session to learn how to use correctly the exercise equipment and how to prevent Valsalva maneuver during the exercise. Initial RT intensity was set to $30 \%$ of 1-RM; then, it was gradually increased to the final $60 \%$ of 1-RM. Each of the RT exercises was always performed in three sets of 10 repetitions each (with 1 min pause between the series). AT was performed regularly three times per week (Monday - Wednesday - Friday) during the same time period from 10:00 to 12:00 AM for the entire period of 12 weeks.

Patients in the EMS group $(n=29)$ underwent transcutaneous EMS of lower limb extensors at home. The quadriceps and calf muscles of both legs were stimulated using adhesive electrodes 80 x 130 mm (PALS ${ }^{\circledR}$ Platinum, Axelgaard, Denmark) and portable battery-powered stimulator (REHAB X-2, Cefar $^{\circledR}$, Malmö, Sweden). EMS was always performed 2 x 60 min per day, 7 day per week for the entire period of 12 weeks. Technical parameters of the stimulation were selected as follows: two-phase electric current with $10 \mathrm{~Hz}$ frequency, in "on-off” operation mode (20 s of contraction, $20 \mathrm{~s}$ of relaxation) and maximal amplitude of $60 \mathrm{~mA}$. The first application of EMS was performed in hospital under medical supervision. After familiarization with the stimulation technique and the correct placement of electrodes, patients continued with the EMS at home. The intensity of stimulation was progressively increased to cause a visible contraction tolerable by the patient (the stimulation intensity during the first 2 weeks was $30-40 \mathrm{~mA}$, and then it was increased to the target level of $60 \mathrm{~mA}$ which was not changed until the end of rehabilitation period). Patients regularly ( $1 \mathrm{x} /$ week) submitted the stimulators and electrodes for technical inspection at the clinic.

Patients in the AT + EMS group $(n=28)$ performed standard bicycle AT according to a protocol identical to that of the AT group. In addition, these patients applied EMS using the technical equipment and stimulation protocol identical to that of the EMS group. EMS was performed at home only on the days without supervised aerobic training on bicycle (4 times a week) for the total period of 12 weeks.

The influence of the RHB program on the quality of life (QoL) was evaluated using a Czech version of the Minnesota Living with Heart Failure (MLHF) questionnaire (21 items). At the end of the rehabilitation period (12 weeks), a new evaluation of all functional parameters and the QoL identical to the initial assessment was carried out.

\section{Statistical data analysis}

The data were analyzed according to Eng ${ }^{18}$. Frequency analysis, estimates of arithmetic mean and standard error were used as summary statistics describing primary data. Prior to any parametric data processing, the normality of sample distribution (Shapiro-Wilk's test) and homogeneity of variance (Levene's test) was verified. Pearson's correlation coefficient was used as a measure of mutual correlation between original parameters as well as measure of correlation between the differences in values due to experimental exercise. ML- $\chi^{2}$ test (categorical variables) and one-way ANOVA model (continuous variables) were applied for comparison of initial patient characteristics among experimental variants. Repeated measure ANOVA model was applied as principal method evaluating outcomes of experiments with model design including experimental groups (AT, EMS, AT+EMS as independent, effect component) and sampling in different time points (as dependent, pair-wise component). Statistical significance of differences among experimental groups was tested as independent component of rmANOVA model. Greenhouse-Geisser correction was applied to correct violation of sphericity assumption in case of testing repeated measures effect. For detailed mutual comparison of experimental variants with control group, Dunnet's post hoc test (independent comparisons) and pair-wise t-test (pair-wise comparisons) were used. Patients were considered as "responders" to exercise training if they reached $>10 \%$ increase in $\mathrm{VO}_{2 \text { peak }}$. Statistical analyses were computed using SPSS 19.0.1 (IBM Corporation, 2010). A value $P<0.05$ was accepted as boundary of statistical significance in all applied tests.

\section{RESULTS}

A total of 86 patients were included in the study. Only 71 of them completed the 12-week rehabilitation training cycle. Evaluation was done for 26 patients in the AT group ( 3 did not complete), 23 in the EMS group (6 did not complete), and 22 in the AT + EMS group (6 did not complete). A total of 15 patients finished the training program prematurely. The reasons for not completing were: loss of motivation ( 7 cases), 6 patients admitted irregular EMS application for a period longer than 1 week, and 2 patients ended RHB due to a change of residence. Patients in the EMS group and those with the combination AT+EMS who completed the entire training program tolerated EMS very well. There were no reports of muscle pains, dyspnea, nausea or dermatological problems caused by the electrodes. There was no statistically significant difference between the three groups in any of the initial characteristics (Table 1).

\section{Functional parameters}

The principal statistical analysis was based on repeated measures ANOVA model and on pair-wise comparison of changes within the experimental groups during 12 weeks of follow-up (Table 2). 
Table 1. The initial characteristics of patients in compared experimental groups.

\begin{tabular}{lcccc}
\hline Parameter $^{1}$ & $\begin{array}{c}\text { AT group } \\
(\mathrm{N}=26)\end{array}$ & $\begin{array}{c}\text { EMS group } \\
(\mathrm{N}=23)\end{array}$ & $\begin{array}{c}\text { AT + EMS group } \\
(\mathrm{N}=22)\end{array}$ & $P^{2}$ \\
\hline Age $($ years $)$ & $63.5(1.4)$ & $57.3(1.6)$ & $60.8(2.5)$ & 0.079 \\
Male sex & $\mathrm{N}=24(92.3 \%)$ & $\mathrm{N}=15(65.2 \%)$ & $\mathrm{N}=17(77.3 \%)$ & 0.227 \\
Weight $(\mathrm{kg})$ & $88.8(2.4)$ & $85.4(2.6)$ & $87.9(3.4)$ & 0.675 \\
Height $(\mathrm{cm})$ & $174.4(1.5)$ & $173.9(1.6)$ & $174.5(2.1)$ & 0.966 \\
NYHA II/III & $19 / 4$ & $18 / 5$ & $18 / 4$ & - \\
EF (\%) & $34.9(1.4)$ & $30.1(1.3)$ & $32.8(1.9)$ & 0.094 \\
BMI & $29.2(0.7)$ & $28.3(0.9)$ & $28.8(0.8)$ & 0.747 \\
Exercise duration $(\min )$ & $8: 21(0: 25)$ & $8: 00(0: 28)$ & $9: 15(0: 32)$ & 0.189 \\
MLHF score & $34.3(3.5)$ & $38.6(4.9)$ & $32.0(4.0)$ & 0.081 \\
HR ${ }_{\text {rest }}$ (beats/min) & $68.5(3)$ & $66.6(4)$ & $65.8(3)$ & 0.214 \\
Systolic BP ${ }_{\text {rest }}$ (mmHg) & $110.2(5.2)$ & $114.9(4.8)$ & $112.8(4.4)$ & 0.303 \\
Diastolic BP ${ }_{\text {rest }}$ (mmHg) & $65.3(4.1)$ & $68.2(2.2)$ & $70.8(5.3)$ & 0.154 \\
\hline Pharmacotherapy & & & & \\
ACE inhibitors & 26 & 23 & 22 & - \\
Beta-blockers & 15 & 16 & 13 & - \\
Diuretics & 14 & 15 & 17 & - \\
Digoxin & 2 & 3 & 3 & - \\
Statins & 20 & 18 & 18 & - \\
\hline CHF etiology & & 16 & 16 & - \\
Coronary artery disease & 21 & 7 & 6 & - \\
Dilated cardiomyopathy & 5 & & & \\
\hline
\end{tabular}

${ }^{1}$ Categorical data are described by absolute number and percentage of patients in given category; continuous variables are described by mean (S.E.) ${ }^{2}$ Statistical significance of differences among groups was tested by ML- $\chi^{2}$ test for categorical data and by one-way ANOVA model for continuous parameters.

Both dependent component (pair-wise changes in time due to exercise) and independent component (main effect of experimental treatment) of variability were tested. Comparing pair-wise reaction on exercise in key endpoints $\left(\mathrm{VO}_{2 \text { peak }}, \mathrm{VO}_{2 \mathrm{AT}}\right.$ and $\left.\mathrm{W}_{\text {peak }}\right)$ revealed statistically significant improvement of patients in all experimental groups. Changes over time in spiroergometric parameters were expressed as percentages of the initial absolute values (due to under- or overestimation of results) and interpreted on the basis of valid recommendations ${ }^{19}$. The changes induced by experimental training were quantitatively comparable in all three groups (averaged difference after 12 weeks of the exercise as related to the initial value: $\Delta \mathrm{VO}_{2 \text { peak }}:+12.9 \%, \Delta \mathrm{VO}_{2 \mathrm{AT}}:+9.3 \%$, and $\left.\Delta \mathrm{W}_{\text {peak }}:+22.7 \%\right)$. As a consequence, no statistically significant difference among experimental groups was found for the main effect component in the 0rmANOVA model. Similar statistically significant pair-wise changes due to all three modes of exercise were observed in the other examined parameters $\left(\mathrm{VE} / \mathrm{VCO}_{2}\right.$ slope and $\left.\mathrm{HR}_{\text {peak }}\right)$, again without statistically significant differences among experimental groups. Furthermore, patients recruited in all three experimental groups significantly improved exercise duration, on average by $+12.9 \%$ as related to the initial value $(P=0.001)$.
At the end of RHB period there were no significant changes in resting values of HR [group AT 68.2(4); group EMS 66.3(3); group AT + EMS 68.8(3) beats/min], systolic BP [group AT 116.2(4.5); group EMS 115.1(5.1); group AT + EMS 117.1(5.4) $\mathrm{mmHg}]$, and diastolic BP [group AT 69.7(4.3); group EMS 71.8(4.1); group AT a EMS 68.2(3.9) $\mathrm{mmHg}$ ] compared to values at baseline. Although the mean changes in all key end-points due to experimental exercise regimes were statistically significant, the variability of responses to the training was increased due to occurrence of some proportion of non-responders. Non-responders in all three experimental groups were defined as patients with $\leq 10 \%$ change in parameter $\mathrm{VO}_{2 \text { peak }}$ of its initial value, calculated over the course of the experiment. There was no statistically significant difference in the occurrence of non-responders among the experimental groups. Table 3 summarizes proportion of non-responders in all examined combinations.

\section{Quality of life score}

MLHF global score was significantly improved in the AT group ( $\triangle$ MLHF: $-27.9 \%$ based on initial value, $P=0.001)$, in the group with combined training AT + EMS ( $\triangle$ MLHF: $-29.1 \%$ based on initial value, $P=0.001$ ), and 
Table 2. The results of experimental testing for 12 weeks in three groups: AT $(N=28)$, EMS $(N=23)$ and AT/EMS $(\mathrm{N}=22)$.

\begin{tabular}{|c|c|c|c|c|c|}
\hline \multirow{2}{*}{$\begin{array}{c}\text { Parameter } \\
\text { Group }\end{array}$} & \multirow{2}{*}{\multicolumn{2}{|c|}{$\begin{array}{c}\text { Experimental results }^{1} \\
\text { Initial point (T0) 12th week (T12) }\end{array}$}} & \multicolumn{2}{|c|}{ Differences: $\mathrm{T} 12-\mathrm{T} 0^{2}$} & \multirow[b]{2}{*}{$P^{4}$} \\
\hline & & & Value & $\%$ of initial values & \\
\hline \multicolumn{6}{|l|}{$\mathrm{VO}_{2 \text { peak }}\left(\mathrm{ml} \cdot \mathrm{kg}^{-1} \cdot \mathrm{min}^{-1}\right)$} \\
\hline $\mathrm{AT}^{\text {pectan }}$ & $17.8(0.9)$ & $20.6(0.9)$ & $2.7(0.7)$ & $15.2 \%$ & 0.001 \\
\hline EMS & $16.9(1.0)$ & $18.4(0.9)$ & $1.4(0.3)$ & $8.3 \%$ & 0.001 \\
\hline $\mathrm{AT}+\mathrm{EMS}$ & $17.8(0.9)$ & $20.5(1.0)$ & $2.7(0.5)$ & $15.3 \%$ & 0.001 \\
\hline$P^{3}$ & \multicolumn{4}{|c|}{0.405} & \\
\hline \multicolumn{6}{|l|}{$\overline{\mathrm{VO}_{2 \mathrm{AT}}\left(\mathrm{ml} \cdot \mathrm{kg}^{-1} \cdot \mathrm{min}^{-1}\right)}$} \\
\hline AT & $11.4(0.5)$ & $12.5(0.7)$ & $1.1(0.6)$ & $9.6 \%$ & 0.043 \\
\hline EMS & $10.9(0.6)$ & $11.6(0.5)$ & $0.7(0.3)$ & $6.4 \%$ & 0.030 \\
\hline $\mathrm{AT}+\mathrm{EMS}$ & $10.9(0.5)$ & $12.1(0.5)$ & $1.3(0.3)$ & $11.9 \%$ & 0.001 \\
\hline$P^{3}$ & \multicolumn{4}{|c|}{0.568} & \\
\hline \multicolumn{6}{|l|}{$\overline{\mathrm{VE} / \mathrm{VCO}_{2} \text { slope }}$} \\
\hline AT & $33.4(1.2)$ & $29.3(0.9)$ & $-4.1(0.9)$ & $-12.3 \%$ & 0.013 \\
\hline EMS & $33.2(1.4)$ & $30.2(0.8)$ & $-3.0(1.1)$ & $-9.0 \%$ & 0.012 \\
\hline $\mathrm{AT}+\mathrm{EMS}$ & $31.4(1.2)$ & $28.5(0.7)$ & $-2.9(1.1)$ & $-9.2 \%$ & 0.016 \\
\hline$P^{3}$ & \multicolumn{4}{|c|}{0.314} & \\
\hline \multicolumn{6}{|l|}{$\mathrm{W}_{\text {peak }}\left(\right.$ Watt.kg $\left.^{-1}\right)$} \\
\hline $\mathrm{AT}^{\text {peak }}$ & $1.2(0.1)$ & $1.4(0.1)$ & $0.3(0.0)$ & $25.0 \%$ & 0.001 \\
\hline EMS & $1.1(0.1)$ & $1.3(0.1)$ & $0.2(0.0)$ & $18.2 \%$ & 0.001 \\
\hline $\mathrm{AT}+\mathrm{EMS}$ & $1.2(0.1)$ & $1.5(0.1)$ & $0.3(0.1)$ & $25.0 \%$ & 0.001 \\
\hline$P^{3}$ & \multicolumn{4}{|c|}{0.367} & \\
\hline \multicolumn{6}{|l|}{$\overline{\mathrm{HR}_{\text {pank }}(\mathrm{bpm})}$} \\
\hline $\mathrm{AT}^{\text {peak }}$ & $116.9(3.3)$ & $128.6(2.7)$ & $11.7(3.1)$ & $10.0 \%$ & 0.001 \\
\hline EMS & $120.0(5.2)$ & $125.9(5.1)$ & $5.9(2.3)$ & $4.9 \%$ & 0.020 \\
\hline AT+EMS & $126.0(4.7)$ & $136.9(4.2)$ & $10.9(1.9)$ & $8.7 \%$ & 0.001 \\
\hline$P^{3}$ & \multicolumn{4}{|c|}{0.233} & \\
\hline \multicolumn{6}{|c|}{ Duration of exercise (min) } \\
\hline AT & $8: 21(0: 25)$ & 10:01 (0:26) & $1: 39(0: 24)$ & $19.8 \%$ & 0.001 \\
\hline EMS & $8: 00(0: 28)$ & $8: 46(0: 30)$ & $0: 45(0: 16)$ & $9.4 \%$ & 0.013 \\
\hline $\mathrm{AT}+\mathrm{EMS}$ & $9: 15(0: 32)$ & $10: 15(0: 28)$ & $1: 00(0: 15)$ & $10.7 \%$ & 0.001 \\
\hline$P^{3}$ & \multicolumn{4}{|c|}{0.119} & \\
\hline \multicolumn{6}{|l|}{ MLHF score } \\
\hline AT & $34.3(3.5)$ & $24.7(3.0)$ & $-9.6(2.1)$ & $-27.9 \%$ & 0.001 \\
\hline EMS & $38.6(4.9)$ & $32.2(4.9)$ & $-6.4(2.1)$ & $-16.6 \%$ & 0.008 \\
\hline $\mathrm{AT}+\mathrm{EMS}$ & $32.0(4.0)$ & $22.7(3.5)$ & $-9.3(1.9)$ & $-29.1 \%$ & 0.002 \\
\hline$P^{3}$ & \multicolumn{4}{|c|}{0.021} & \\
\hline
\end{tabular}

${ }^{1}$ Arithmetic mean and standard error (SE)

${ }^{2}$ Pair-wise differences expressed as difference of arithmetic means (standard error) and as \% of initial value

${ }^{3}$ Significance level of independent component in rm ANOVA model

${ }^{4}$ Significance level of pair-wise (time-related) component in rm ANOVA model

also in EMS group ( $\triangle$ MLHF: $-16.6 \%$ based on initial value, $P=0.008$ ). Analyzing the final values after the exercise, the EMS group was significantly different from AT group (final MLHF score was 24.7) and AT + EMS (final MLHF score 22.7; $P=0.021$ ).

\section{DISCUSSION}

Supervised ET performed in the context of a cardiovascular RHB program is traditionally considered to be an integral part of the overall therapeutic strategy for
CHF management. Improvement in functional capacity and QoL, in particular, are the main positives ${ }^{20}$. The maintenance of physical fitness is a strong prognostic factor of $\mathrm{CHF}$, whereas reduced functional capacity, loss of muscular strength and inactivity are (often underestimated) the main risk factors ${ }^{21}$. Combined interval training (aerobic + resistance elements) is generally recommended - its higher effectiveness in CHF patients has been proven, especially improving $\mathrm{VO}_{2 \text { peak }}$ and left ventricular function $^{22,23}$. The results of this study do not deviate from the generally good experience with the positive influence of aerobic exercise training on the health status of CHF pa- 
tients ${ }^{10,24}$. The resulting improvement in $\mathrm{VO}_{2 \text { peak }}$ peak values in the groups with bicycle training (AT and AT+EMS) averaged $+15.2 \%$, which corresponds with previously published experience from extensive clinical studies stating average improvement between $+10 \%$ and $+26 \%$ (ref. ${ }^{19}$ ) or $+21 \%$ (ref. $^{25}$ ). In the present study, a total of 38 (i.e. ca $54 \%$ ) of the 71 patients who completed RHB program were evaluated as "responders", i.e. showing $\geq 10 \% \mathrm{im}$ provement in $\mathrm{VO}_{2 \text { peak }}$. Also this finding conforms to the data from the literature ${ }^{10}$. It is therefore evident that a majority of CHF patients reacted positively to the 12-week training by increasing values of $\mathrm{VO}_{2 \text { peak }}$ as well as of $\mathrm{VO}_{2 \mathrm{AT}}$ and $\mathrm{W}_{\text {peak }}$. The percentage occurrence of "non-responders" $\left(\mathrm{VO}_{2 \text { peak }}^{\text {peak }} \leq 10 \%\right)$ in the groups with bicycle training (AT and AT + EMS) ranged around $41 \%$, on average, while in the EMS group it was approx. 57\%; this finding corresponds also to the rather modest increase in $\Delta \mathrm{VO}_{2 \text { peak }}$ (only $+8.3 \%$ in EMS group). The higher number of "nonresponders" in the EMS group might indicate lower effectiveness when using of EMS only. However, it is important that not in single case of a "non-responder" significant deterioration in $\mathrm{VO}_{2 \text { peak }}$ output values was observed. The $\mathrm{VE} / \mathrm{VCO}_{2}$ slope parameter has been recently considered as a very important prognostic factor in $\mathrm{CHF}$ patients ${ }^{17,26,27}$. The relationship between $\mathrm{VE}$ and $\mathrm{VCO}_{2}$ expresses ventilation effectiveness during exercise, i.e. records the amount of ventilated air which is necessary to evacuate $1 \mathrm{~L}$ of $\mathrm{CO}_{2}$. During exercise, $\mathrm{VE}$ and $\mathrm{VCO}_{2}$ increase linearly until reaching RER, when the increase of VE sharply rises in comparison to $\mathrm{VCO}_{2}$ (ref. ${ }^{28}$ ). In $\mathrm{CHF}$ patients, the $\mathrm{VE} / \mathrm{VCO}_{2}$ slope value increases - while normal values range between 20 and 30, in $\mathrm{CHF}$ patients they rise to as high as 80 (ref. ${ }^{19}$ ). $\mathrm{VE} / \mathrm{VCO}_{2}$ can be positively affected by aerobic training and - as shown - it represents greater prognostic value than $\mathrm{VO}_{2 \text { peak }}\left(\right.$ ref. $^{29-31}$ ). In the present study, there was a significant decrease of $\mathrm{VE} /$ $\mathrm{VCO}_{2}$ slope in all three experimental groups, by $-10.2 \%$ after RHB program. In absolute numbers there was a decrease in the $\mathrm{VE} / \mathrm{VCO}_{2}$ slope to below 30 in both groups with AT, while in the EMS group the final value was borderline $^{30}$. Overall, this outcome can be considered a very positive shift from the perspective of prognosis for development of the disease; moreover, the influence of all three types of RHB protocol was comparable. The functional parameters improvement in the EMS group was fully comparable to those of the groups with aerobic training. This may seem surprising, if one considers the more or less local effects of EMS (only thigh and calf muscles). However, according to previously published findings, significant improvement of performance can be achieved even with local muscle exercise, and it is comparable to the traditional "full-body" training ${ }^{32,33}$. Therefore, EMS conforms to the criteria for achieving highly intensive muscle work in the stimulated muscles. Quality of life evaluation is among the primary indicators as to a training program's effectiveness and should be a part of any clinical study for CHF patients. The average decrease in the total MLHF score across all three groups was $-24.6 \%$ (statistically significant in all groups). However, MLHF scoring was the only parameter where the rmANOVA model recognized significant differences among experimental groups. The smallest, time-related improvement (and thus the highest level of the final MLHF score) after 12 weeks of exercise was observed in the EMS group (final score 32.2). Despite this, the overall result achieved can be considered a positive impact of regular muscle exercise on the QoL in CHF patients. This is in concordance with previously published experiences ${ }^{34,35}$. The physiological response to the increase of oxygen demand during contraction caused by EMS is similar to that caused by normal physical exercise. Banerjee et al. ${ }^{36}$ observed in a group of sedentary, healthy people that $\mathrm{VO}_{2 \text { pea }}$ increased by a significant $+10 \%$ and there was overall improvement in functional performance after 6-week EMS. In the present study, the improvement in $\mathrm{VO}_{2 \text { peak }} \mathrm{EMS}$ group was somewhat lower $(+8.3 \%)$ but still statistically significant to that for the other groups. In the past decade, several studies focused on the EMS application in CHF. In a pilot study, Maillefert et al. ${ }^{37}$ studied 14 patients with CHF. After 5 weeks of EMS $(10 \mathrm{~Hz})$, the authors observed an increase of $\mathrm{VO}_{2 \text { peak }}(+14 \%)$ and $\mathrm{VO}_{2 \mathrm{AT}}(+23.6 \%)$. In a randomized study, Nuhr et al. ${ }^{38}$ compared the effects of EMS with a control group. The protocol included $4 \mathrm{~h}$ of direct stimulation of both quadriceps muscles daily (15 $\mathrm{Hz}$ ); EMS was applied 7 days weekly for 10 weeks. The authors found a significant increase of $\mathrm{VO}_{2 \text { peak }}, \mathrm{VO}_{2 \mathrm{AT}}$ and increase in walked distance during a 6-min walking test in stimulated group. Muscle biopsy in this study showed increased citrate synthase activity, decreased glyceraldehyde-phosphate dehydrogenase activity, and increase of myosin heavy chain isoforms. Another randomized study from 2005, 24 patients were divided into 2 groups - group with AT and EMS group ${ }^{39}$. The stimulation protocol included EMS $(10 \mathrm{~Hz})$ of quadriceps and triceps surae muscles. A nearly identical improvement in $\mathrm{VO}_{2 \text { peak }}(+11 \%)$ and $\mathrm{VO}_{2 \mathrm{AT}}(+17 \%)$ was observed in both groups. Finally, in a study from 2009, Banerjee et al. ${ }^{40}$ recorded a significant improvement of approx. $+10 \%$ in $\mathrm{VO}_{2 \text { eak }}$ in $\mathrm{CHF}$ patients due to EMS. Muscle stimulation is accepted as one of the traditional methods of rehabilitation and physical medicine. There are several advantages in EMS and moreover its safety has been clinically proven. It can be used for patients for whom the standard physical activity cannot be prescribed due to the severity of disease and presence of comorbidities ${ }^{41}$. EMS causes no undesirable changes in hemodynamic parameters, its overall tolerance is good and patients do not complain of muscle pain, nausea or skin problems. EMS improves the functional characteristics of skeletal muscles not only in healthy volunteers $^{42}$ or post-injury situations ${ }^{43}$ - its therapeutic effects have been already used for affecting several chronic diseases or for post-operative treatment in intensive care units $^{44}$. From a physiological point of view, the final differentiation of mammalian muscle fibers into their individual subtypes is directly dependent on the electrical activity parameters of the driving motoneurons. EMS simulates the electrical activity of the driving motoneurons; setting up external electrical impulses at the appropriate frequency can substitute the original natural activity and as a result this promotes a number of func- 
tional and metabolic changes in the muscle fibers ${ }^{45,46}$. At first glance, muscular contractions evoked by EMS have similar acute effects on the neuromuscular system as voluntary muscular contractions (VMC), but during VMC motor units (MU) are activated synchronously in the direction from small to large depending on the intensity of physical workload ${ }^{47}$. EMS, however, by-passes the physiological hierarchy and the external electrical impulses activate all MU at the same time. Thus, the muscle work of maximal intensity can be achieved almost immediately. Despite the differences mentioned, EMS and VMC in combination can be considered as two mutually supporting stimuli of a different character and which, with a view to the longer term (e.g. as parts of an RHB program), might influence the resulting effectiveness of training both from the qualitative and quantitative perspectives ${ }^{48}$. In a 2008 study Paillard ${ }^{49}$ demonstrated in detail the muscular adaptations with a long-term combination of EMS + AT (or EMS only) in healthy individuals, active sportsmen and patients after knee surgery. Combined training had greater efficacy than voluntary exercise alone, which was interpreted as a cumulative effect in the processes of muscular adaptations. In the context of RHB, it was also shown that EMS was an ideal complement to voluntary exercise. In the early stage of RHB it increased muscular strength, necessary for carrying out the classical training activities. In our study, the AT + EMS group had a higher rate of improvement in the absolute values of those parameters which are generally considered to be main criteria for evaluating the effectiveness of rehabilitation - $\mathrm{VO}_{2 \mathrm{p}}$ and $\mathrm{VO}_{2 \mathrm{AT}}$ (an improvement of maximal and submaximal performance). This might be seen as confirmation of the study presumption that the greatest training benefit was expected to result from the combination of AT and EMS. Statistical evaluation, however, did not confirm significant differences between the three groups. In other words, all three training methods had approximately the same positive effect on the functional performance and QoL of CHF patients.

\section{Study limitations}

There are several reasons which might explain the fact that greater benefit from AT + EMS combination was not objectively demonstrated in our study. First, it must be taken into account that a large percentage of those studies in which the combination of classical training and EMS brought greater benefit were done in patients after orthopedic and surgical interventions or entirely healthy individuals and active sportsmen. The pathophysiology of the functional and metabolic changes in $\mathrm{CHF}$ is so complex that the resulting effect of the combined training need not significantly have manifested itself. Another question relates to the stimulation protocol used. Although the $10 \mathrm{~Hz}$ frequency is well-established in clinical practice, it cannot be ruled out that a higher rate of improving performance might be achieved using other frequencies (e.g. 25, 35, or $50 \mathrm{~Hz}$ ). Another factor which could significantly affect the final results was the number of patients undertaking the AT + EMS combination (only 22 persons from the original 28 completed the training). Finally, the authors were limited in their possibility to check-up the frequency and regularity of EMS application at home. The only sources of information were the stimulated patients themselves; only 6 patients admitted irregular use of EMS (leading to their elimination from the study). These limitations do not, however, in any way decrease the significance of EMS which should be regarded as a very attractive and effective method of cardiovascular rehabilitation. The use of EMS, however, is still sporadic, even though it has proven to be very useful in improving the functional RHB outcomes in $\mathrm{CHF}$ patients.

\section{CONCLUSION}

The main aim of this study was to evaluate the effectiveness of combination of classical ET and EMS in patients with a less advanced form of CHF. The results confirmed the performance improvement using EMS alone or its combination with standard exercise training in patients with heart failure. However, the results of the three studied RHB training protocols did not differ statistically significantly. It can be stated that aerobic ET combined with EMS adds no statistically significant benefit. Nevertheless, a very important positive fact needs to be emphasized - all the studied training methods improved the parameters of functional fitness and quality of life in $\mathrm{CHF}$ patients. Further research is needed in order to determine the optimal protocol of EMS and its combinations with other exercise training techniques in the rehabilitation of heart failure.

\section{CONFLICT OF INTEREST STATEMENT}

The authors stated that there are no conflicts of interest regarding the publication of this article.

\section{ACKNOWLEDGEMENT}

Supported by the grant IGA MZCR NS 100096/4.

\section{REFERENCES}

1. Dickstein K, Cohen-Solal A, Filippatos G, McMurray JJ, Ponikowski P, Poole-Wilson PA, Strömberg A, van Veldhuisen DJ, Atar D, Hoes AW, Keren A, Mebazaa A, Nieminen M, Priori SG, Swedberg K; ESC Committee for Practice Guidelines (CPG). ESC guidelines for the diagnosis and treatment of acute and chronic heart failure 2008: the Task Force for the diagnosis and treatment of acute and chronic heart failure 2008 of the European Society of Cardiology. Developed in collaboration with the Heart Failure Association of the ESC (HFA) and endorsed by the European Society of Intensive Care Medicine (ESICM). Eur J Heart Fail 2008;10:933-89.

2. Böhm M, Kilter H, Kindermann M. Mechanisms contributing to the progression of left ventricular dysfunction to end-stage heart failure. Eur Heart J 2003;5:114-21.

3. Hobbs FD, Davis RC, Lip GY. ABC of heart failure: Heart failure in general practice. BMJ 2000;320:626-9.

4. Mancini DM, Walter G, Reichek N, Lenkinski R, McCully KK, Mullen JL, Wilson JR. Contribution of skeletal muscle atrophy to exercise intol- 
erance and altered muscle metabolism in heart failure. Circulation 1992;85:1364-73.

5. Clark A, Rafferty D, Arbuthnott K. Relationship between isokinetic muscle strength and exercise capacity in chronic heart failure. Int J Cardiol 1997;59:145-8.

6. Stanley WC, Chandler MP. Energy metabolism in the normal and failing heart: potential for therapeutic interventions. Heart Fail Rev 2002;7:115-30.

7. Schulze PC, Linke A, Schoene N, Winkler SM, Adams V, Conradi S, Busse M, Schuler G, Hambrecht R. Functional and morphological skeletal muscle abnormalities correlate with reduced electromyographic activity in chronic heart failure. Eur J Cardiovasc Prev Rehabil 2004;11:155-61.

8. Williams AD, Selig S, Hare DL, Hayes A, Krum H, Patterson J, Geerling $\mathrm{RH}$, Toia D, Carey MF. Reduced exercise tolerance in CHF may be related to factors other than impaired skeletal muscle oxidative capacity. J Card Fail 2004;10:141-8.

9. Swedberg K, Cleland J, Dargie H, Drexler H, Follath F, Komajda M, Tavazzi L, Smiseth OA, Gavazzi A, Haverich A, Hoes A, Jaarsma T, Korewicki J, Lévy S, Linde C, Lopez-Sendon JL, Nieminen MS, Piérard $\mathrm{L}$, Remme WJ; Task Force for the Diagnosis and Treatment of Chronic Heart Failure of the European Society of Cardiology. Guidelines for the diagnosis and treatment of chronic heart failure: executive summary (update 2005): The Task Force for the Diagnosis and Treatment of Chronic Heart Failure of the European Society of Cardiology. Eur Heart J 2005;26:1115-40.

10. European Heart Failure Training Group. Experience from controlled trials of physical training in chronic heart failure. Protocol and patient factors in effectiveness in the improvement in exercise tolerance. Eur Heart J 1998;19:466-75.

11. Keteyian SJ, Levine AB, Brawner CA, Kataoka T, Rogers FJ, Schairer $J R$, Stein PD, Levine TB, Goldstein S. Exercise training in patients with heart failure. A randomized, controlled trial. Ann Intern Med, 1996;124:1051-57.

12. Kiilavuori K, Näveri H, Salmi T, Härkönen M.The effect of physical training on skeletal muscle in patients with chronic heart failure. Eur J Heart Fail 2000;2:53-63.

13. Vaquero AF, Chicharro JL, Gil L, Ruiz MP, Sánchez V, Lucía A, Urrea S, Gómez MA. Effects of muscle electrical stimulation on peak $\mathrm{VO}_{2}$ in cardiac transplant patients. Int J Sports Med. 1998;19:317-22.

14. Dobsak P, Novakova M, Siegelova J, Fiser B, Vitovec J, Nagasaka M, Kohzuki M, Yambe T, Nitta S, Eicher JC, Wolf JE, Imachi K. Lowfrequency electrical stimulation increases muscle strength and improves blood supply in patients with chronic heart failure. Circ J 2006;70:75-82.

15. Delitto A, McKowen JM, McCarthy JA, Shively RA, Rose SJ. Electrically elicited co-contraction of thigh musculature after anterior cruciate ligament surgery: a description and single-case experiment. Physical Therapy 1988;68:45-50.

16. Walls RJ, McHugh G, O'Gorman DJ, Moyna NM, O'Byrne JM. Effects of preoperative neuromuscular electrical stimulation on quadriceps strength and functional recovery in total knee arthroplasty; a pilot study. BMC Musculoskeletal Disorders 2010;11:119.

17. Wasserman K, Hansen JE and Sue DY. Principles of exercise testing and interpretation: Pathophysiology and clinical applications. $4^{\text {th }}$ ed. Philadelphia: Lippincott Williams \& Wilkins, 2005.

18. Eng J. ROC analysis: web-based calculator for ROC curves. Baltimore: Johns Hopkins University [updated 2006 May 17; cited 2008 February 20]. Available from: http://www.jrocfit.org.

19. Task Force of the Italian Working Group on Cardiac Rehabilitation and Prevention (Gruppo Italiano di Cardiologia Riabilitativa e Prevenzione, GICR); Working Group on Cardiac Rehabilitation and Exercise Physiology of the European Society of Cardiology. Statement on cardiopulmonary exercise testing in chronic heart failure due to left ventricular dysfunction: recommendations for performance and interpretation Part III: Interpretation of cardiopulmonary exercise testing in chronic heart failure and future applications. Eur J Cardiovasc Prev Rehabil 2006;13:485-94.

20. Davies EJ, Moxham T, Rees K, Singh S, Coats AJ, Ebrahim S, Lough F, Taylor RS. Exercise training for systolic heart failure: Cochrane systematic review and meta-analysis. Eur J Heart Fail 2010;12:706-15.

21. Larsen AI, Lindal S, Aukrust P, Toft I, Aarsland T, Dickstein K. Effect of exercise training on skeletal muscle fibre characteristics in men with chronic heart failure. Correlation between skeletal muscle alterations, cytokines and exercise capacity. Int J Cardiol 2002;83:25-32.
22. Wisløff U, Støylen A, Loennechen JP, Bruvold M, Rognmo Ø, Haram PM, Tjønna AE, Helgerud J, Slørdahl SA, Lee SJ, Videm V, Bye A, Smith GL, Najjar SM, Ellingsen $\varnothing$, Skjaerpe T. Superior cardiovascular effect of aerobic interval training versus moderate continuous training in heart failure patients: a randomized study. Circulation 2007;115:3086-94.

23. Bouchla A, Karatzanos E, Dimopoulos S, Tasoulis A, Agapitou V, Diakos N, Tseliou E, Terrovitis J, Nanas S. The addition of strength training to aerobic interval training: effects on muscle strength and body composition in CHF patients. J Cardiopulm Rehabil Prev 2011;31:47-51.

24. Piepoli MF, Davos C, Francis DP, Coats AJ; ExTraMATCH Collaborative. Exercise training meta-analysis of trials in patients with chronic heart failure (ExTraMATCH). BMJ 2004;328:189-98.

25. Keteyian SJ, Fleg JL, Brawner CA, Piña IL. Role and benefits of exercise in the management of patients with heart failure. Heart Fail Rev 2010;15:523-30.

26. Jaussaud J, Aimable L, Douard $H$. The time for a new strong functional parameter in heart failure: the VE/VCO2 slope. Int J Cardiol. 2011;147:189-90.

27. Van Laethem C, Van De Veire N, De Backer G, Bihija S, Seghers T, Cambier D, Vanderheyden M, De Sutter J. Response of the oxygen uptake efficiency slope to exercise training in patients with chronic heart failure. Eur J Heart Fail 2007;9:625-9.

28. Mezzani A, Agostoni $P$, Cohen-Solal A, Corrà U, Jegier A, Kouidi E, Mazic S, Meurin P, Piepoli M, Simon A, Laethem CV, Vanhees L. Standards for the use of cardiopulmonary exercise testing for the functional evaluation of cardiac patients: a report from the Exercise Physiology Section of the European Association for Cardiovascular Prevention and Rehabilitation. Eur J Cardiovasc Prev Rehabil 2009;16:249-67.

29. Van de Veire NR, Van Laethem C, Philippé J, De Winter O, De Backer G, Vanderheyden M, De Sutter J. VE/VCO2 slope and oxygen uptake efficiency slope in patients with coronary artery disease and intermediate peak VO . Eur J Cardiovasc Prev Rehabil 2006;13:916-23.

30. Ingle L, Goode K, Carroll S, Sloan R, Boyes C, Cleland JG, Clark AL. Prognostic value of the VE/VCO2 slope calculated from different time intervals in patients with suspected heart failure. Int J Cardiol 2007; 118:350-5.

31. Myers J, Arena R, Oliveira RB, Bensimhon D, Hsu L, Chase P, Guazzi M, Brubaker P, Moore B, Kitzman D, Peberdy MA. The lowest VE/VCO2 ratio during exercise as a predictor of outcomes in patients with heart failure. J Card Fail 2009;15:756-62.

32. Magnusson G, Kaijser L, Sylvén C, Karlberg K-E, Isberg B, Saltin B. Peak skeletal muscle perfusion is maintained in patients with chronic heart failure when only a small muscle mass is exercised. Cardiovasc Res 1997;33:297-306.

33. Gordon A, Tyni-Lenné R, Jansson E, Kaijser L, Theodorsson-Norheim E, Sylvén C. Improved ventilation and decreased sympathetic stress in chronic heart failure patients following local endurance training with leg muscles. J Cardiac Failure 1997;3:3-12.

34. Willenheimer R, Erhardt L, Cline C, Rydberg E, Israelsson B. Exercise training in heart failure improves quality of life and exercise capacity. Eur Heart J 1998;19:774-81.

35. O'Connor CM, Whellan DJ, Lee KL, Keteyian SJ, Cooper LS, Ellis SJ, Leifer ES, Kraus WE, Kitzman DW, Blumenthal JA, Rendall DS, Miller $\mathrm{NH}$, Fleg JL, Schulman KA, McKelvie RS, Zannad F, Piña IL; HF-ACTION Investigators. Efficacy and safety of exercise training in patients with chronic heart failure: HF-ACTION randomized controlled trial. JAMA 2009;301:1439-50.

36. Banerjee $P$, Caulfield B, Crowe L, Clark A. Prolonged electrical muscle stimulation exercise improves strength and aerobic capacity in healthy sedentary adults. Journal of Applied Physiology 2005;99:2307-11.

37. Maillefert JF, Eicher JC, Walker P, Dulieu V, Rouhier-Marcer I, Branly F, Cohen M, Brunotte F, Wolf JE, Casillas JM, Didier JP. Effects of low-frequency electrical stimulation of quadriceps and calf muscles in patients with chronic heart failure. J Cardiopulm Rehabil 1998; 18:277-82.

38. Nuhr MJ, Pette D, Berger R, Quittan M, Crevenna R, Huelsman M, Wiesinger GF, Moser P, Fialka-Moser V, Pacher R. Beneficial effects of chronic low-frequency stimulation of thigh muscles in patients with advanced chronic heart failure. Eur Heart J 2004;25:136-43.

39. Deley G, Kervio G, Verges B, Hannequin A, Petitdant MF, SalmiBelmihoub S, Grassi B, Casillas JM. Comparison of low-frequency 
electrical myostimulation and conventional aerobic exercise training in patients with chronic heart failure. Eur J Cardiovasc Prev Rehabil 2005; 12:226-33.

40. Banerjee P, Caulfield B, Crowe L, Clark AL. Prolonged electrical muscle stimulation exercise improves strength, peak $\mathrm{VO}_{2}$, and exercise capacity in patients with stable chronic heart failure. J Card Fail 2009;15:319-26.

41. Wiesinger GF, Crevenna R, Nuhr MJ, Huelsmann M, Fialka-Moser V Quittan M. Neuromuscular electric stimulation in heart transplantation candidates with cardiac pacemakers. Arch Phys Med Rehabi 2001;82:1476-7.

42. Pérez M, Lucia A, Santalla A, Chicharro JL. Effects of electrical stimulation on $\mathrm{VO}_{2}$ kinetics and delta efficiency in healthy young men. $\mathrm{Br} J$ Sports Med 2003;37:140-3.

43. Hainaut K, Duchateau J.Neuromuscular electrical stimulation and voluntary exercise. Sports Med 1992;14:100-13.

44. Strasser EM, Stättner S, Karner J, Klimpfinger M, Freynhofer M, Zaller V, Graf A, Wessner B, Bachl N, Roth E, Quittan M. Neuromuscular electrical stimulation reduces skeletal muscle protein degradation and stimulates insulin-like growth factors in an age- and currentdependent manner: a randomized, controlled clinical trial in major abdominal surgical patients. Ann Surg 2009;249:738-43.

45. Pette $D$, Vrbova $G$. What does chronic electrical stimulation teach us about muscle plasticity? Muscle Nerve 1999;22:666-77.

46. Ljubicic V, Adhihetty P, Hood D. Application of animal models: chronic electrical stimulation-induced contractile activity. Can J Appl Physiol 2005;30:625-43.

47. Sanchez BR, Puche PP, Gonzame-Badillo JJ. Percutaneous electrical stimulation in strength training: an update. J Strength Cond Res 2005;19:438-48.

48. Miller C, Thepaut-Mathieu C. Strength training by electrostimulation conditions for efficacy. Int J Sports Med 1993;14:20-8.

49. Paillard T. Combined application of neuromuscular electrical stimulation and voluntary muscular contractions. Sports Med 2008;38:16177. 